\title{
IMPACT OF PELVIC GYNECOLOGICAL CANCER ON FEMALE SEXUALITY
}

\author{
M. Bouhani1, O. jaidane1, W. homri2, M. slimene1, M.A. bouida1, J. ben hassouna1, M. \\ hechiche1, R. chargui1, R. labbene2, K. rahal1 \\ 1Salah Azaiz Institute, Oncologic Surgery, Tunis, Tunisia; \\ 2Razi hospital, department $C$ of psychiatry, Tunis, Tunisia
}

\section{Objectives:}

Gynaecological malignancies represent $11.3 \%$ of cancers in women in Tunisia.

Currently, cancer is considered a chronic disease. Assessing the impact of cancer occurrence and its treatment on female sexuality often involves communication difficulties between the patient and the health care team.

The purpose of this work was to:

-Determine female sexual dysfunction in patients treated for pelvic gynecologic cancer.

-Plan an early sexual rehabilitation program for such patients

\section{Methods:}

This was a cross-sectional descriptive study that was conducted over a four-month period, from February 1st, 2017 to June 30th, 2017 at Salah Azaiez Institute.

\section{Results:}

The median age was 47.6 years. Half of our patients were housewives. The illiteracy rate was $36.7 \%$. The average duration of the marriage was 26.7 years. Cervical cancer was the most common type (40\%). Chemotherapy was performed in $60 \%$ of patients, while radiotherapy was performed in $70 \%$ of patients. Stage II was the most common (70\%).

The different clinical data on gynaecological cancer are summarized in Table I.

Table I. Characteristics of cancer disease

\begin{tabular}{|llll|}
\hline Cancer type & Number (\%) & Stage & Treatment \\
\hline Cervix cancer & $12(40 \%)$ & Stage I : 3 & RT + CHL \\
& & $\begin{array}{l}\text { Stage II : 8 } \\
\text { Stage III : 1 }\end{array}$ & RT + RT + CT +CHL \\
\hline Endometrial cancer & $8(26,7 \%)$ & Stage I : 4 & CHL \\
& & Stage II : 4 & CHL + RT + CT \\
\hline Ovarian cancer & $5(16,7 \%)$ & Stage I : 1 & Conservative ovarian \\
& & Stage II : 4 & staging + CT \\
& & & Complete ovarian staging + \\
& & & CT \\
\hline Vulvar cancer & $4(13,3 \%)$ & Stage II : 3 & Vulvectomy + bilateral \\
& & Stage III : 1 & inguinal lymph node \\
& & & dissection+ RT \\
\hline Vulvar cancer & $1(3,3 \%)$ & Stage I : 1 & Partial vaginectomy + RT \\
\hline
\end{tabular}

$\mathrm{CHL}$ : colpohysterectomy + bilateral lymphadenectomy, RT: radiotherapy

CT: chemotherapy, ovarian staging: hysterectomy + adnexectomy + pelvic and lumbo-aortic lymph node dissection + omentectomy.

Patients already had sexual dysfunction prior to cancer diagnosis with Female Sexual Function Index (FSFI) of 25 (Table 2). Impairment in sexual function affecting all components of the FSFI index was observed with a statistically significant $p$. Predictors of sexual dysfunction after gynecologic cancer were age, level of education, duration of marriage, occupation, type of disease, and administration of chemotherapy.
Table 2. The FSFI score according to its different items before and after a cancer diagnosis

\begin{tabular}{|c|c|c|c|c|c|c|c|}
\hline \multirow{2}{*}{$\begin{array}{l}\text { Items } \\
\text { 1. Desire : frequency }\end{array}$} & \multirow{2}{*}{$\begin{array}{l}\begin{array}{l}\text { Score before } \\
\text { (min-max) }\end{array} \\
2-4\end{array}$} & \multirow{2}{*}{$\begin{array}{l}\text { Score after } \\
\text { (min-max) }\end{array}$} & \multicolumn{2}{|c|}{$\begin{array}{l}\text { Average } \\
\text { before }\end{array}$} & \multicolumn{2}{|c|}{ Average after } & \multirow{2}{*}{$\begin{array}{l}P \\
0.009\end{array}$} \\
\hline & & & 3.3 & \multirow[t]{2}{*}{4.1} & 1.9 & \multirow[t]{2}{*}{2.4} & \\
\hline 2. Desire : level & $2-5$ & 1-4 & 3.6 & & 2.2 & & \\
\hline 3. Arousal : frequency & $2-5$ & $0-4$ & 3.5 & \multirow[t]{4}{*}{4.1} & 2.3 & \multirow[t]{4}{*}{2.7} & \multirow[t]{4}{*}{0.001} \\
\hline 4. Arousal : level & $1-5$ & $0-4$ & 3.4 & & 2.3 & & \\
\hline 5. Arousal : confidence & $1-5$ & $0-4$ & 3.4 & & 2.3 & & \\
\hline 6. Arousal : satisfaction & $1-5$ & $0-4$ & 3.6 & & 2.2 & & \\
\hline 7. Lubrication : frequency & $1-5$ & $0-3$ & 3.4 & \multirow[t]{4}{*}{4} & 1.5 & \multirow[t]{4}{*}{1.5} & \multirow[t]{4}{*}{0.039} \\
\hline 8. Lubrication : difficulty & $1-5$ & $0-3$ & 3.4 & & 1.2 & & \\
\hline 9. Lubrication : frequency of maintaining & $1-5$ & $0-3$ & 3.5 & & 1.4 & & \\
\hline 10. Lubrication: the difficulty of maintaining & $1-5$ & $0-2$ & 3.1 & & 1 & & \\
\hline 11. Orgasm : frequency & $1-5$ & $0-3$ & 3.2 & \multirow[t]{3}{*}{4.1} & 1.8 & \multirow[t]{3}{*}{2.2} & \multirow[t]{3}{*}{0.004} \\
\hline 12. Orgasm : difficulty & $1-5$ & $0-3$ & 3.4 & & 2 & & \\
\hline 13. Orgasm : satisfaction & $1-5$ & $0-3$ & 3.7 & & 1.7 & & \\
\hline $\begin{array}{l}\text { 14. Satisfaction with amount of closeness } \\
\text { with partner }\end{array}$ & $1-5$ & $0-4$ & 3.4 & \multirow[t]{3}{*}{4.4} & 2.7 & \multirow[t]{3}{*}{2.8} & \multirow[t]{3}{*}{0.005} \\
\hline 15. Satisfaction with sexual Relationship & $2-5$ & $1-3$ & 3.8 & & 2.1 & & \\
\hline 16. Satisfaction with overall sex life & 3-5 & $1-3$ & 4 & & 2.2 & & \\
\hline $\begin{array}{l}\text { 17. Pain: Frequency during vaginal } \\
\text { penetration }\end{array}$ & $1-5$ & $0-3$ & 3.6 & \multirow[t]{3}{*}{4.3} & 1.7 & \multirow[t]{3}{*}{2} & \multirow[t]{3}{*}{0.013} \\
\hline 18. Pain: Frequency after vaginal penetration & $2-5$ & $0-3$ & 3.6 & & 1.7 & & \\
\hline $\begin{array}{l}\text { 19. Pain: Level during or after vaginal } \\
\text { penetration }\end{array}$ & $1-5$ & $0-3$ & 3.7 & & 1.6 & & \\
\hline TOTAL & & & & 25 & & 13.6 & 0.0008 \\
\hline
\end{tabular}

\section{Conclusions:}

Sexuality after cancer affecting the female genital area is altered, age $>50$ years, illiteracy, length of marriage $>$ 20 years as well as endometrial, vulva and vaginal cancers were predictive factors of sexual dysfunction. 